\title{
Associação entre aves e flores de duas espécies de árvores do gênero Erythrina (Fabaceae) na Mata Atlântica do sudeste do Brasil
}

\author{
Ricardo Parrini ${ }^{1} \&$ Marcos A. Raposo ${ }^{2}$ \\ 1. Rua Desembargador Isidro, 126, bloco C, apto. 801, 20521-160 Rio de Janeiro, RJ, Brasil. \\ 2. Setor de Ornitologia, Departamento de Vertebrados, Museu Nacional/UFRJ, Quinta da Boa Vista s/n, 20940-040 Rio de Janeiro, RJ, \\ Brasil. (raposo@mn.ufrj.br)
}

\begin{abstract}
Association between birds and flowers of two species of trees of the genus Erythrina (Fabaceae) in the Atlantic forest of southeastern Brazil. Flowers are considered important resources in the diet of birds during the dry season. We studied the interaction between birds and two species of the trees of genus Erythrina (E. falcata and E. verna) at four localities in the state of Rio de Janeiro: Serra dos Órgãos, Serra da Mantiqueira, Vale do Paraíba, and the southern coastal area (Costa Verde). Sampling was during the dry season. The purpose of this study was to identify the bird species recorded visiting these Leguminosae species and to describe their feeding behavior. A total of 27 bird species were recorded feeding on nectar and 16 were recorded catching arthropods attracted by the flowers. Some strategies used by birds to exploit nectar are described. The presence of so many species and the time spent by them on the trees corroborate the importance of nectar consumption and flowers to the bird community during the dry season. The importance of bird species on pollination is also discussed.
\end{abstract}

KEYWORDS. Erythrina, birds, flower resources, pollination.

RESUMO. Flores são consideradas um importante recurso na alimentação de aves em épocas secas. A interação entre aves e árvores do gênero Erythrina (E. falcata e E. verna) foi estudada em quatro regiões do Estado do Rio de Janeiro: Serra dos Órgãos, Serra da Mantiqueira, Vale do Paraíba e região litorânea da Costa Verde. A amostragem foi realizada na época mais seca do ano. Objetivou-se listar as espécies de aves que utilizam recursos florais e descrever os comportamentos associados. Ao todo, 27 espécies de aves foram observadas alimentando-se de néctar ou outros recursos florais e 16 apenas predando artrópodes atraídos pelas flores. A presença de um número elevado de espécies e o tempo despendido por elas nas árvores corroboram a relevância do néctar e das florações como recursos alimentares para a comunidade de aves na época mais seca do ano. É discutido também o papel exercido pelas aves na polinização.

PALAVRAS-CHAVE. Erythrina, aves, recursos florais, polinização.

Erythrina (Fabaceae) é um gênero pantropical com cerca de 110 espécies, tendo sua polinização efetuada principalmente por aves (BRUNEAU, 1997; COTTON, 2001). A interação entre aves e plantas desse gênero foi estudada na Região Neotropical por MORTON (1979), FEINSINGER et al. (1979), Cotton (2001) e Ragusa-Netto (2002), entre outros. Alguns trabalhos empreendidos em distintas regiões do Brasil mostraram a relevância das florações de diferentes espécies do gênero Erythrina na alimentação das aves. Vitali-Veiga \& Machado (2000) constataram que as flores de Erythrina speciosa constituem importante fonte alimentar para as aves no inverno, em área urbana da cidade de Rio Claro, Estado de São Paulo. Ragusa-Netto (2002), por sua vez, revelou que a floração de Erythrina dominguezii representa recurso alimentar alternativo para aves frugívoras em florestas secas do oeste do Brasil durante os meses da estação seca. Nesse sentido, alguns autores, como TERBORGH (1986) e Olmos \& Boulhosa (2000), sugeriram que plantas que possuem alta produção de frutos ou flores podem atrair muitas espécies de aves e mamíferos em épocas de escassez de chuvas, funcionando como recursos-chave. Na Mata Atlântica, alguns trabalhos têm relatado a associação entre aves e plantas do gênero Erythrina (Vitali-Veiga \& Machado, 2000; Almeida \& Alves, 2001; WiLlis, 2002).

Este estudo enfoca as aves observadas consumindo os recursos florais de Erythrina falcata e
Erythrina verna em quatro diferentes regiões do Estado do Rio de Janeiro, durante a estação mais seca de diferentes anos. Os objetivos foram determinar as espécies de aves que se alimentam desses recursos florais e descrever os seus comportamentos. Discute-se com base nos dados levantados a importância das flores de E. falcata e E. verna como recurso alimentar para as aves, assim como potenciais deslocamentos empreendidos pelas aves e o papel destas como agentes polinizadores.

\section{MATERIAL E MÉTODOS}

Erythrina falcata é uma árvore de grande porte, com até $35 \mathrm{~m}$ de altura, que ocorre em vários países da América do Sul: Argentina, Bolívia, Paraguai, Peru e Brasil (CARVAlho, 2003). No Brasil, ocorre desde Minas Gerais e Mato Grosso do Sul até o Rio Grande do Sul. É encontrada com regularidade em formações secundárias e capoeiras, sendo típica para o sopé das grandes serras "com neblina" (CARvalho, 2003). Suas flores são vermelhas ou alaranjadas, de 3 a $5 \mathrm{~cm}$ de comprimento, situadas em numerosos cachos pendentes na extremidade de ramos. No Estado do Rio de Janeiro, a espécie floresce em agosto e setembro (CARVALHO, 2003).

Erythrina verna é uma árvore de até $20 \mathrm{~m}$ de altura que ocorre na Bolívia e no Brasil, sendo encontrada em várias regiões, incluindo o sudeste brasileiro (BACKES \& 
IRGANG, 2004). Assim como a espécie anterior, é encontrada principalmente em formações secundárias. Suas flores são vermelhas, tendo até $5 \mathrm{~cm}$ de comprimento, situadas em cachos pendentes na extremidade de ramos. A floração ocorre em agosto e setembro, com a árvore desprovida de folhas (BACKES \& IRGANG, 2004). No gênero Erythrina, o néctar é acumulado no cálice, na base das flores (VITALIVeiga \& Machado, 2000; CotTon, 2001).

$\mathrm{O}$ presente estudo foi empreendido em quatro diferentes regiões do Rio de Janeiro onde a vegetação original, a Floresta Pluvial Atlântica, ainda pode ser encontrada, especialmente em parques nacionais e na região da Costa Verde (litoral sul do estado), onde mesclase com áreas antrópicas em diversos setores.

As florações de E. falcata foram acompanhadas na Serra dos Órgãos e da Mantiqueira e as de E. verna no Vale do Paraíba e na região litorânea da Costa Verde.

Na Serra dos Órgãos, as observações foram feitas no município de Teresópolis ( $22^{\circ} 24^{\prime} \mathrm{S}, 42^{\circ} 57^{\prime} \mathrm{W}$ ) em duas localidades distintas: no Parque Nacional da Serra dos Órgãos (altitudes entre 800 e $1.400 \mathrm{~m}$ ) e no Bairro de Ermitage $(820 \mathrm{~m})$, nas seguintes datas: 31.05 .2001 , 06.06.2001, 18 a 20.06.2001, 01.07.2002, 05.07.2002, 9 e 10.07.2002. Foram observadas 32 árvores, tanto em bordas de florestas quanto em locais abertos, totalizando 960min de observação.

Na Serra da Mantiqueira, as observações foram feitas no município de Itatiaia ( $\left.22^{\circ} 29^{\prime} \mathrm{S}, 4^{\circ} 33^{\prime} \mathrm{W}\right)$ nos limites do Parque Nacional de Itatiaia (altitudes entre 750 e 1.420m), nas seguintes datas: 09 a 13.06.2001, 27.07.2002, 29.07.2002, 18 a 20.08.2002, 18 a 21.08.2003, 10.07.2004, 12.07.2004, 17 e 18.07.2004, 17 a 20.08.2004, 12 e 13.06.2005. Foram observadas 29 árvores, a maioria delas situada em bordas de florestas, totalizando 1.750min de observação.

No Vale do Paraíba, as observações foram empreendidas no município de Barra Mansa (2232’S, $44^{\circ} 10^{\prime} \mathrm{W}$ ), ao longo da estrada RJ-155, entre a cidade de Barra Mansa e a localidade de Getulândia (altitudes entre 420 e $500 \mathrm{~m}$ ), nas seguintes datas: 20 e 21.10 .2001 e 29 e 30.07.2002. Ao longo dos dez quilômetros iniciais dessa estrada, 17 árvores foram observadas, sempre em locais abertos e capoeiras, totalizando $420 \mathrm{~min}$ de observação.

Na região da Costa Verde, as observações foram empreendidas em duas localidades: os arredores da localidade de Perequê (altitudes de 30 a 50m), no município de Angra dos Reis (230' $\mathrm{S}, 44^{\circ} 19^{\prime} \mathrm{W}$ ), em 13 e 14.09 .2001 e 20 e 21.10 .2004 , e no município de Parati $\left(23^{\circ} 13^{\prime} \mathrm{S}\right.$, $44^{\circ} 42^{\prime} \mathrm{W}$ ), em área da Fazenda Olaria situada a $2 \mathrm{~km}$ ao sul da cidade, na margem da estrada BR-101 (altitude de 30m), entre 08 e 10.11.1995. Foram observadas 23 árvores em Angra dos Reis e 17 em Parati, em orla de florestas e locais abertos, totalizando 830min de observação.

As estações de floração observadas para E. falcata e E. verna foram mais longas do que as citadas na literatura por CARVALHO (2003) e BACKES \& IRGANG (2004), mas ainda dentro da época de estio na Região Sudeste (final de abril a meados de novembro) (DAvIs, 1945).

Foram utilizados dois métodos para observar as aves em Erythrina: o método de árvore-focal e o de transecto. No primeiro, observou-se uma determinada árvore por períodos de até $2 \mathrm{~h}$ pela manhã, sempre até 09:00h, anotando-se as espécies que consumiam recursos florais ou artrópodes, assim como seus comportamentos. No segundo, utilizado em apenas alguns dias, percorreram-se trilhas ou estradas, anotando-se o número de árvores, espécies de aves e comportamentos associados. Foram utilizados binóculos 8x30 nas observações.

A identificação das duas espécies vegetais foi feita mediante comparação direta nos herbários do Parque Nacional da Serra dos Órgãos e do Jardim Botânico do Rio de Janeiro. A nomenclatura utilizada para as aves foi a de SicK (1997).

\section{RESULTADOS}

Ao todo 27 espécies de aves foram observadas alimentando-se de recursos florais de Erythrina spp. A tabela I mostra as espécies de aves observadas, o número de registros em cada região, o método alimentar e o padrão das visitas (número de indivíduos) exibido por cada espécie. A seguir são enumerados detalhes e variações comportamentais observados nas quatro diferentes famílias de aves identificadas.

Cracidae. Penelope obscura (Temminck, 1815) foi observada em bandos com 4 a 9 indivíduos em E. falcata, sempre comendo brotos de flores ainda fechadas no início da floração.

Psittacidae. As espécies dessa família foram registradas geralmente em bandos com até 22 indivíduos, apresentando comportamentos de certa forma semelhantes entre si. Aratinga leucophthalmus (Statius Muller, 1776), em grupos de até 18 indivíduos, utilizou em similar proporção dois tipos de comportamentos. No primeiro, as aves inseriram o bico nas flores para sorver o néctar, parecendo em alguns casos trincar e furar a base da flor. No outro método, as aves arrancaram as flores com o bico e mandibularam-nas de forma a sorver o néctar, sendo a flor descartada sob a árvore ao final do processo. Neste último caso, o tempo gasto desde a remoção até o momento em que a flor era descartada variou de 3 a 10 s. Uma vez que a flor é descartada, a ave logo se move nos ramos para coletar outra flor. Brotogeris tirica (Gmelin, 1788), em bandos de até 16 indivíduos, alternou comportamento similar ao primeiro mencionado para $A$. leucophthalmus com outro, em que as aves removeram as flores com o bico e em seguida utilizaram um dos pés para segurá-las enquanto sorviam o néctar. Observamos aves removendo de 4 a 7 flores em um minuto. Este último comportamento foi exibido também por Brotogeris chiriri (Vieillot, 1818) e Pyrrhura frontalis (Vieillot, 1817), sendo mais freqüentemente observado nesta última espécie. Pyrrhura frontalis, que empreendeu visitas com até 22 indivíduos, empregou, adicionalmente, os mesmos comportamentos mencionados para A. leucophthalmus em que não ocorre a utilização dos pés. Pionus maximiliani (Kuhl, 1820), em bandos de até 12 indivíduos, sempre removeu as flores utilizando eventualmente as patas.

Embora não tenhamos observado o consumo de pétalas por membros dessa família, achamos possível a ingestão, mesmo que involuntária, e sobretudo de outras partes florais (e. g. estames) durante a mandibulação empreendida por algumas espécies. 
Visitas com duração de aproximadamente 30 a 40min foram registradas para A. leucophthalmus $(\mathrm{n}=3)$, B. tirica $(\mathrm{n}=7)$ e $P$. frontalis $(\mathrm{n}=5)$. Para esta última espécie, foram registradas outras com tempo superior a $140 \mathrm{~min}(\mathrm{n}=2)$. Durante o tempo em que as aves permaneceram nas árvores ocorreram, no entanto, períodos de inatividade em que as aves estiveram pousadas sem se alimentar.

Trochilidae. Várias espécies de beija-flores utilizaram os recursos florais de Erythrina. Para obtenção de néctar, as espécies pairaram ou pousaram brevemente nas pétalas enquanto inseriam o bico na flor. Entre as espécies observadas, Eupetomena macroura (Gmelin, 1788), Clytolaema rubricauda (Boddaert, 1783) e Leucochloris albicollis (Vieillot, 1818) apresentaram comportamentos territoriais, espantando outras espécies de beija-flores. Leucochloris albicollis, aparentemente a mais territorial entre elas, perseguiu indivíduos da própria espécie $(\mathrm{n}=14)$ e de outras, como C. rubricauda $(\mathrm{n}=3)$ e Stephanoxis lalandi (Vieillot, 1818) $(\mathrm{n}=1)$. Clytolaema rubricauda foi a espécie de ave mais registrada em E. falcata na Serra dos Órgãos, onde foi observada em agregações de até cinco indivíduos.
Leucochloris albicollis, em grupos de até dez indivíduos, foi uma das espécies mais freqüentemente encontradas em E. falcata, tanto na Serra dos Órgãos como na Mantiqueira. Outras espécies que empreenderam visitas em grupos foram Colibri serrirostris (Vieillot, 1816), com até seis indivíduos, e Anthracothorax nigricollis (Vieillot, 1817), com até 13 indivíduos em E. verna. Eupetomena macroura foi registrada em pequenos grupos de 3 a 4 indivíduos e apenas em E. verna. As demais espécies empreenderam visitas com até dois indivíduos. Phaethornis eurynome (Lesson, 1832), S. lalandi e Aphantochroa cirrhochloris (Vieillot, 1818) foram visitantes raros de E. falcata.

Algumas espécies de beija-flor estiveram entre as aves com as visitas mais prolongadas. Esse é o caso de L. albicollis e E. macroura, as quais permaneceram por mais de $120 \mathrm{~min}$ em E. falcata, e de A. nigricollis, com períodos de 40 a $60 \mathrm{~min}$ em E. verna. Assim como os psitacídeos, os beija-flores também "descansaram" em certos períodos durante estas longas estadias nas árvores.

Emberizidae. Essa família revelou diversidade comportamental durante a obtenção de recursos florais,

Tabela I. Número e características das visitas de aves que utilizaram recursos florais de Erythrina spp. (Fabaceae) em quatro áreas amostradas no Estado do Rio de Janeiro, sudeste do Brasil. Recurso floral explorado: Pe, pétalas; Ne, néctar; Br, brotos de flor. Método utilizado para obtenção do recurso alimentar: A, inserir o bico entre as pétalas para obtenção de néctar; B, perfurar a base da flor para obtenção de néctar; C, remover flores inteiras com o bico para sorver néctar; $\mathrm{D}$, remover e ingerir pedaços de pétalas; E, remover e ingerir brotos de flores. Padrão das visitas: S, ave solitária; P, em pares; B, em bando.

\begin{tabular}{|c|c|c|c|c|c|c|c|}
\hline \multicolumn{3}{|c|}{ Erythrina falcata } & \multicolumn{2}{|c|}{ Erythrina verna } & \multirow[b]{2}{*}{$\begin{array}{c}\text { Recurso } \\
\text { explorado }\end{array}$} & \multirow[b]{2}{*}{$\begin{array}{l}\text { Método de } \\
\text { obtenção }\end{array}$} & \multirow[b]{2}{*}{$\begin{array}{c}\text { Padrão das } \\
\text { visitas }\end{array}$} \\
\hline $\begin{array}{l}\text { Famílias e } \\
\text { espécies de aves }\end{array}$ & $\begin{array}{l}\text { Serra dos } \\
\text { Órgãos }\end{array}$ & $\begin{array}{c}\text { Serra da } \\
\text { Mantiqueira }\end{array}$ & $\begin{array}{l}\text { Vale do } \\
\text { Paraíba }\end{array}$ & Costa Verde & & & \\
\hline \multicolumn{8}{|l|}{ CRACIDAE } \\
\hline Penelope obscura & & 5 & & & $\mathrm{Br}$ & $\mathrm{E}$ & $\mathrm{B}$ \\
\hline \multicolumn{8}{|l|}{ PSITTACIDAE } \\
\hline Aratinga leucophthalmus & 4 & 2 & 6 & & $\mathrm{Ne}$ & $\mathrm{A}, \mathrm{C}$ & $\mathrm{P}, \mathrm{B}$ \\
\hline Pyrrhura frontalis & 7 & 21 & & & $\mathrm{Ne}$ & $\mathrm{A}, \mathrm{C}$ & $\mathrm{P}, \mathrm{B}$ \\
\hline Brotogeris tirica & 8 & 2 & & 7 & $\mathrm{Ne}$ & $\mathrm{A}, \mathrm{C}$ & $\mathrm{P}, \mathrm{B}$ \\
\hline Brotogeris chiriri & & 4 & & & $\mathrm{Ne}$ & $\mathrm{C}$ & $\mathrm{P}, \mathrm{B}$ \\
\hline Pionus maximiliani & 3 & & & & $\mathrm{Ne}$ & $\mathrm{C}$ & $\mathrm{P}$ \\
\hline \multicolumn{8}{|l|}{ TROCHILIDAE } \\
\hline Phaethornis eurynome & 2 & & & & $\mathrm{Ne}$ & A & S \\
\hline Eupetomena macroura & 9 & 3 & 7 & & $\mathrm{Ne}$ & A & $\mathrm{S}, \mathrm{P}, \mathrm{B}$ \\
\hline Melanotrochilus fuscus & 8 & & 2 & 16 & $\mathrm{Ne}$ & A & $\mathrm{S}, \mathrm{P}$ \\
\hline Colibri serrirostris & & & 5 & 4 & $\mathrm{Ne}$ & A & $\mathrm{S}, \mathrm{P}, \mathrm{B}$ \\
\hline Anthracothorax nigricollis & & & 2 & 11 & $\mathrm{Ne}$ & A & $\mathrm{S}, \mathrm{P}, \mathrm{B}$ \\
\hline Stephanoxis lalandi & 1 & 1 & & & $\mathrm{Ne}$ & A & $\mathrm{S}$ \\
\hline Thalurania glaucopis & 16 & 10 & 3 & & $\mathrm{Ne}$ & A & $\mathrm{S}, \mathrm{P}$ \\
\hline Leucochloris albicollis & 32 & 17 & 1 & 3 & $\mathrm{Ne}$ & A & $\mathrm{S}, \mathrm{P}, \mathrm{B}$ \\
\hline Amazilia versicolor & 6 & 2 & 6 & 9 & $\mathrm{Ne}$ & A & $\mathrm{S}, \mathrm{P}$ \\
\hline Amazilia lactea & & & 5 & & $\mathrm{Ne}$ & A & $\mathrm{S}, \mathrm{P}$ \\
\hline Aphantochroa cirrhochloris & 1 & & & & $\mathrm{Ne}$ & A & $\mathrm{S}$ \\
\hline Clytolaema rubricauda & 37 & 6 & & & $\mathrm{Ne}$ & A & $\mathrm{S}, \mathrm{P}, \mathrm{B}$ \\
\hline \multicolumn{8}{|l|}{ EMBERIZIDAE } \\
\hline Coereba flaveola & 11 & 5 & 6 & 6 & $\mathrm{Ne}$ & $\mathrm{A}, \mathrm{B}$ & $\mathrm{S}, \mathrm{P}, \mathrm{B}$ \\
\hline Cissopis leveriana & & 3 & & & $\mathrm{Br}$ & E & $\mathrm{P}$ \\
\hline Ramphocelus bresilius & & & 3 & 5 & $\mathrm{Ne}$ & A & $\mathrm{S}, \mathrm{P}$ \\
\hline Thraupis sayaca & 2 & 3 & 3 & 6 & $\mathrm{Ne}-\mathrm{Pe}$ & A, D & $\mathrm{S}, \mathrm{P}, \mathrm{B}$ \\
\hline Tangara cayana & 1 & & & & $\mathrm{Ne}$ & A & $\mathrm{P}$ \\
\hline Dacnis nigripes & & 4 & & & $\mathrm{Ne}$ & A & $\mathrm{P}, \mathrm{B}$ \\
\hline Dacnis cayana & 14 & 18 & 9 & 5 & $\mathrm{Ne}$ & A & $\mathrm{S}, \mathrm{P}, \mathrm{B}$ \\
\hline Psarocolius decumanus & 4 & & & 2 & $\mathrm{Ne}$ & A & B \\
\hline Cacicus haemorrhous & & 33 & & 3 & $\mathrm{Ne}$ & $\mathrm{A}, \mathrm{C}$ & $\mathrm{P}, \mathrm{B}$ \\
\hline
\end{tabular}


com uso freqüente da técnica do "espaçar" (SICK, 1997). Cacicus haemorrhous (Linnaeus, 1766) foi a espécie de ave com maior número de registros (n=33) em $E$. falcata na Serra da Mantiqueira, tendo sido registrada poucas vezes na Costa Verde, em E. verna. O número de indivíduos por visita variou de 2 a 32. No dia 12.06.2001, entre 06:20 e 06:40 h, no P. N. de Itatiaia, registramos aproximadamente 32 indivíduos durante intensa atividade em torno de diferentes cachos de flores de uma E. falcata. Três tipos diferentes de comportamentos para obter néctar foram utilizados por C. haemorrhous, ocorrendo a remoção de flores em apenas um deles. O mais comum consiste na ave introduzir o bico por entre as pétalas para sorver o néctar $(\mathrm{n}=32)$, de forma semelhante ao observado para Psarocolius decumanus (Pallas, 1769). No segundo mais utilizado, a ave arranca a flor com o bico e a leva até um galho horizontal próximo para então firmá-la com um dos pés sobre o ramo e introduzir o bico por entre as pétalas $(\mathrm{n}=18)$. No terceiro, a ave inicialmente puxa a flor com o bico e, a seguir, segura-a com um dos pés enquanto insere o bico por entre as pétalas sem arrancar a flor de seu ramo. O último comportamento foi observado apenas em E. falcata. A técnica do "espaçar" foi registrada durante os três comportamentos citados anteriormente, no momento em que as aves inseriam o bico nas flores $(\mathrm{n}=16)$.

Cissopis leveriana (Gmelin, 1788) comeu pequenos botões novos e ainda fechados de flores com coloração marrom-avermelhada. Coereba flaveola (Linnaeus, 1788) obteve néctar de duas maneiras. Na primeira, a ave, enquanto inseria o bico por entre pétalas de flores ainda fechadas, utilizou o "espaçar" de forma a abrir espaço para sorver o néctar. Na outra, aves perfuraram a base das flores. Dacnis cayana (Linnaeus, 1766), registrada em todas as regiões, foi uma das espécies mais observadas em flores de E. falcata. Sempre em número de 1 a 4 indivíduos, esta espécie foi observada inserindo o bico em flores abertas e fechadas, sendo que nestas últimas utilizou a técnica do "espaçar" como mencionado para $C$. flaveola. Dacnis nigripes (Pelzeln, 1856) foi observada sempre associada a $D$. cayana e utilizando comportamentos similares. Thraupis sayaca (Linnaeus, 1766), em bandos de até sete indivíduos, removeu e "mascou" pedaços das pétalas de flores ou inseriu o bico entre as pétalas para sorver o néctar, usando também a técnica do "espaçar".

Alguns emberizídeos, como D. cayana e T. sayaca, alimentaram-se também de artrópodes coletados por entre as inflorescências. Tempos de permanência em árvores acima de 30min foram assinalados para C. flaveola, $D$. cayana e Cacicus haemorrhous.

Além do consumo direto dos recursos florais, 15 espécies de aves foram observadas somente capturando artrópodes em E. falcata: Macropsalis creagra (Nitzsch, 1840), Picumnus cirratus (Temminck, 1825), Cranioleuca pallida (Wied, 1831), Philydor rufus (Vieillot, 1818), Sittasomus griseicapillus (Vieillot, 1818), Lepidocolaptes squamatus (Lichtenstein, 1822), Phyllomyias fasciatus (Thunberg, 1822), Myiornis auricularis (Vieillot, 1818), Knipolegus cyanirostris (Vieillot, 1818), Oxyruncus cristatus (Swainson, 1821), Orchesticus abeillei (Lesson, 1839), Hemithraupis ruficapilla (Vieillot, 1818), Tangara seledon (Station Muller, 1776), Tangara cyanoventris (Vieillot, 1819), Thraupis ornata (Sparrman, 1789); e apenas uma em E. verna: Colonia colonus (Vieillot, 1818). As presas foram capturadas em galhos, folhas, no espaço aéreo ou nos cachos de flores.

\section{DISCUSSÃO}

Este estudo reforça o uso intensivo de flores de plantas do gênero Erythrina por diversas espécies de aves na Floresta Atlântica. Em um gradiente altitudinal que se estende desde as florestas costeiras do litoral sul do Estado do Rio de Janeiro (Costa Verde) até as florestas montanas dos Parques Nacionais da Serra dos Órgãos e Itatiaia $(1.420 \mathrm{~m})$, diversas espécies de aves foram observadas consumindo recursos florais de E. verna e E. falcata ao longo da estação mais seca do ano. Assim como relatado em trabalhos anteriores (MORTON, 1979; Cotton, 2001; RAgusa-Neto, 2002), o néctar pode ser visto como recurso alternativo para aves frugívoras das famílias Psittacidae e Emberizidae.

A permanência de várias espécies de aves, amiúde em bandos numerosos, durante longos períodos nas árvores estudadas demonstra que as aves estão optando por explorar por mais tempo os abundantes recursos florais destas plantas ao invés de procurar outras fontes de alimento. Esse foi o caso de alguns beija-flores, como Eupetomena macroura, Leucochloris albicollis e Clytolaema rubricauda, que permaneceram pelo menos durante 2 h de certas manhãs em ramos de E. falcata; de alguns psitacídeos, como Aratinga leucophthalmus e Pyrrhura frontalis, igualmente com longas visitas (40min) pelas manhãs, e de emberizídeos, como Dacnis cayana e Cacicus haemorrhous, observados durante visitas com pelo menos 30min de duração. CоттоN (2001) relatou o mesmo fenômeno em Erythrina fusca, em que algumas espécies da família Psittacidae permaneceram durante as primeiras horas do dia sem abandonar as árvores, mesmo quando não estavam forrageando. Nesse sentido, ressalta-se que a maior parte das aves forragearam nas primeiras horas do dia, como já relatado por CotTon (2001) para E. fusca e por RAGUSA-NETTO (2002) para Erythrina dominguezii.

Comportamentos territoriais exibidos por algumas espécies de aves, sobretudo beija-flores, sugerem que elas estavam defendendo um importante recurso alimentar. Trochilidae, não surpreendentemente, teve mais representantes em Erythrina spp. do que qualquer outra família (12 espécies, $47 \%$ do total). Conforme CоттоN (2001), beija-flores foram observados freqüentemente alimentando-se de néctar em E. fusca e podem ser dependentes desse recurso. Ainda segundo este autor, os beija-flores estabelecem territórios em E. fusca ao longo do dia, parecendo defender uma importante fonte energética.

VIEIRA et al. (1992) sugeriram que a presença de algumas espécies de aves em sua área de estudo poderia estar relacionada com o período de floração de Mabea fistulifera (Euphorbiaceae). Adicionalmente, MoRTon (1979) relatou que o emberizídeo Icterus spurius (Linnaeus, 1766) foi a espécie mais comum em sua área de estudo durante o período de floração de E. fusca. 
É possível que os registros de Brotogeris chiriri e Dacnis nigripes, espécies não mencionadas em trabalhos que descrevem a avifauna do P. N. de Itatiaia (HoLt, 1928; BARTH, 1957; PINTO, 1951, 1954; PARKER \& GoERCK, 1997), estejam associados à floração de E. falcata naquela região.

Particularmente para as aves, as espécies do gênero Erythrina devem ser facilmente localizadas durante deslocamentos sazonais, pelos seguintes motivos: são árvores altas desprovidas de folhagem durante a floração, fato que realça a cor vermelha intensa dos diversos cachos de flores; são geralmente comuns em suas respectivas regiões e, além disso, possuem "distribuição agregada" em certos locais. Altas concentrações de E. falcata e de E. verna podem ser observadas, por exemplo, no P. N. de Itatiaia e em torno da cidade de Barra Mansa, respectivamente.

Erythrina verna e E. falcata, por sua vez, devem beneficiar-se da presença das diversas espécies de aves devido ao já conhecido papel exercido por esse grupo como polinizador de plantas desse gênero (KRUKOOF \& Barneby, 1974; Bruneau, 1997; CotTon 2001; RagusaNetTo, 2002). Alguns trabalhos anteriores, como os de KRUKOFF \& BARNEBY (1974) e TOLEDO \& HERNÁNDEZ (1979), relataram que existem dois padrões básicos de estrutura floral no gênero Erythrina: o primeiro com estrutura ou formato tubular e o segundo não tubular. Ainda conforme esses autores, enquanto espécies de Erythrina com estruturas florais tubulares são polinizadas principalmente por beija-flores (Trochilidae), as de estruturas não tubulares, como é o caso de E. falcata e E. verna, são polinizadas por vários tipos de aves, incluindo também os beija-flores. No presente estudo, a ocorrência de várias espécies das famílias Psittacidae, Trochilidae e Emberizidae consumindo recursos florais das duas espécies de Erythrina corrobora o padrão "generalista" estabelecido por esses autores para estas plantas. Com resultados semelhantes, CоттоN (2001) relatou várias espécies dessas mesmas famílias como potenciais polinizadoras de $E$. fusca, espécie com estrutura nãotubular de flores.

Algumas espécies de aves registradas neste trabalho foram citadas anteriormente como potenciais polinizadoras de outras espécies do gênero Erythrina, também com estruturas florais não tubulares: Anthracothorax nigricollis para E. fusca (CоTтоN, 2001), Coereba flaveola e Dacnis cayana para Erythrina poeppigiana (FeInsinger et al., 1979) e Psarocolius decumanus para $E$. fusca e E. dominguezii (CоттоN, 2001; Ragusa-Netto, 2002).

Conforme ressaltado anteriormente por alguns autores, como CotTon (2001) e RAGUSA-NetTo (2002), aves que inserem o bico (e a cabeça) por entre as pétalas para sorver o néctar são potenciais polinizadoras de plantas do gênero Erythrina, visto que podem, eventualmente, contactar as anteras e estigmas. Nesse sentido, vários membros das famílias Trochilidae e Emberizidae (Coerebinae, Thraupinae, Icterinae) exibiram tal comportamento e, assim, podem estar entre os principais polinizadores das duas espécies de Erythrina aqui estudadas.

Mesmo alguns emberizídeos como Thraupis sayaca e Cacicus haemorrhous, que eventualmente comeram ou removeram flores, mostraram, alternativamente, tais comportamentos e também estão entre os potenciais agentes polinizadores. Os "tanagers" (Emberizidae: Thraupinae) foram citados como potenciais polinizadores de E. fusca por CotTon (2001) e Feinsinger et al. (1979) e de E. poeppigiana pelo último autor.

De outro modo, algumas aves como Penelope obscura e Cissopis leveriana, que foram observadas apenas comendo botões de flores, não devem contribuir à polinização.

Um caso à parte são os membros da família Psittacidae que, freqüentemente, apresentaram comportamentos destrutivos enquanto consumiam recursos florais. Duas espécies dessa família, Brotogeris chiriri e Pionus maximiliani, sempre que observados, apenas arrancavam flores. Cabe ressaltar que RAGusANetTo (2002) relatou que $B$. chiriri atuou como predador de flores em E. dominguezii, em florestas secas do oeste brasileiro.

Aratinga leucophthalmus, Pyrrhura frontalis e Brotogeris tirica, no entanto, apesar de exibirem comportamentos destrutivos na maioria dos casos, algumas vezes inseriram o bico entre as pétalas para sorver o néctar, aparentemente sem destruir partes florais, podendo dessa forma contribuir para a polinização.

No caso das espécies insetívoras, a sua associação com as árvores estudadas ocorre meramente devido à concentração de insetos nas proximidades de cachos com flores. A abundância de artrópodes, especialmente abelhas e vespas (Hymenoptera), percevejos (Hemiptera), besouros (Coleoptera) e borboletas (Lepidoptera), já foi relatada por VITALI-VeIgA \& MACHADO (2000) em Erythrina speciosa e por FeInsinger et al. (1979) em E. fusca e E. poeppigiana.

O conhecimento sobre a relação entre aves e as diferentes espécies vegetais é de vital importância em termos científicos e de conservação. Este estudo descreveu comportamentos e levantou dados que possibilitam o estabelecimento de hipóteses de associação merecedoras de futuros testes. A relevância das florações para a alimentação tanto de espécies de aves nectarívoras (e. g. beija-flores) como frugívoras e onívoras, como também a relação dessas florações com deslocamentos migratórios, são temas quase obrigatórios para futuros estudos sobre a história natural das comunidades de aves do sudeste do Brasil.

Agradecimentos. Somos gratos à Fundação Carlos Chagas Filho de Amparo à Pesquisa do Estado do Rio de Janeiro (FAPERJ), pelo constante suporte ao Setor de Ornitologia, na figura de M. A. Raposo (proc. Instalação E-26/170.871/2003 e Primeiros Projetos E-26/170.642/2004). Somos gratos também a Carlos Rodrigo Meireles Abreu e Renata Stopiglia pelas sugestões e correções do texto, a Jorge Bruno Nacinovic pela correção do "abstract" e a José Fernando Pacheco por importantes informações prestadas aos autores.

\section{REFERÊNCIAS BIBLIOGRÁFICAS}

Almeida, E. M. \& Alves, M. A. S. 2001. Visitação floral por beijaflores relacionada à produção de néctar em Erythrina speciosa Andrews (Fabaceae) em Mata Atlântica, Ilha Grande, RJ. In: Prezoto, F. ed. Anais de Etologia - XIX Congresso Brasileiro de Etologia. Juiz de Fora, Universidade Federal de Juiz de Fora. p.143. 
Backes, P. \& Irgang, B. 2004. Mata Atlântica. As árvores e a paisagem. Porto Alegre, Paisagem do Sul. 396p.

Barth, R. 1957. A fauna do Parque Nacional do Itatiaia. Boletim do Parque Nacional de Itatiaia 6:90-123.

BrunEaU, A. 1997. Evolution and homology of birds pollinated syndromes in Erythrina (Leguminosae). American Journal of Botany 84:54-71.

Carvalho, P. E. R. 2003. Espécies arbóreas brasileiras. v.1. Colombo, EMBRAPA. 1.039p.

Cotton, P. A. 2001. The behavior and interactions of birds visiting Erythrina fusca flowers in the Colombian Amazon. Biotropica 33:662-669.

DAvis, D. E. 1945. The annual cycle of plants, mosquitoes, birds and mammals in two Brazilian forests. Ecological Monographs 15:243-95.

Feinsinger, P.; Linhart, Y. B.; Swarm, L. A. \& Wolfe, J. A. 1979. Aspects of the pollination biology of three Erythrina species on Trinidad and Tobago. Annals of the Missouri Botanical Garden 66:451-471.

Holt, E. 1928. An ornithological survey of the serra do Itatiaia, Brazil. Bulletin of the American Museum of Natural History 57:251-326.

Krukoff, B. A. \& Barneby, R. C. 1974. A conspectus of the genus Erythrina. Lloydia 37:332-459.

Morton, E. S. 1979. Effective pollination of Erythrina fusca by the Orchard Oriole (Icterus spurius): Coevolved behavioral manipulation? Annals of the Missouri Botanical Garden 66:482-489.

Olmos, F. \& Boulhosa R. L. P. 2000. A meeting of opportunists: birds and other visitors to Mabea fistulifera (Euphorbiaceae) inflorescences. Ararajuba 8:93-98.
PArker, T. A. III \& Goerck, J. M. 1997. The importance of national parks and biological reserves to bird conservation in the Atlantic forest region of Brazil. In: Remsen JR., J. V. ed. Studies in Neotropical Ornithology honoring Ted Parker. Washington, American Ornithologists' Union. p. 527-541 (Ornithological Monographs, 48).

Pinto, O. M. O. 1951. Aves do Itatiaia - Lista remissiva e novas achegas à avifauna da região. Papéis Avulsos Dep. Zool. São Paulo 10:155-208.

1954. Aves do Itatiaia. Lista remissiva e novas achegas à avifauna da região. Boletim do Parque Nacional de Itatiaia 3: $1-87$.

Ragusa-Netto, J. 2002. Exploitation of Erythrina dominguezii Hassl. (Fabaceae) nectar by perching birds in a dry forest in western Brazil. Brazilian Journal of Biology 62:877-883.

Sick, H. 1997. Ornitologia brasileira. Rio de Janeiro, Nova Fronteira.

Terborgh, J. 1986. Keystone plant resources in the tropica forest. In: Soulé, M. E. ed. Conservation Biology. Massachussetts, Sinauer. p.330-344.

TOLEDO, V. M. \& HeRnÁNDEZ, H. M. 1979. Erythrina oliviae: a new case of oriole pollination in Mexico. Annals of the Missouri Botanical Garden 66:503-511.

Vieira, M. F.; Matos, G. T. \& Carvalho-Okano, R. M. 1992. Mabea fistulifera (Euphorbiaceae) na alimentação de aves na região de Viçosa, Minas Gerais, Brasil. Iheringia, Série Zoologia, 73:65-68

Vitali-Veiga, M. J. \& Machado, V. L. L. 2000. Visitantes florais de Erythrina speciosa Andr. (Leguminosae). Revista Brasileira de Zoologia 17:369-383.

Willis, E. O. 2002. Birds at Eucalyptus and other flowers in Southern Brazil: a review. Ararajuba 10(1):43-66.

Recebido em agosto de 2005. Aceito em julho de 2007. ISSN 0073-4721

Artigo disponível em: www.scielo.br/isz 